\title{
A NOTE ON USING GRAPHS IN REGULAR SCHEDULING PROBLEMS
}

This paper deals with regular permutation scheduling on graphs. Peško and Czimmermann introduced this problem (in [3]) and it is generalisation of a matrix permutation problem. The goal is to minimise differences between row sums of a real matrix that represents a schedule, but external conditions don't allow moving matrix elements arbitrarily. The conditions can be represented by permutation obtained from a certain graph.

\section{Introduction}

The matrix permutation problem (MPP) is to minimise differences between row sums by permuting elements in columns of a matrix. This problem was defined first by Š. Peško in [5]. The exact definition of MPP (from [1]) is:

There is given matrix $\left(a_{i, j}\right) \in R^{m \times n}$ (we will call it scheduling matrix). We need to find permutations of the numbers $1, \ldots, m$, $\pi_{j}=\left(\pi_{j}(1), \ldots, \pi_{j}(i)\right)$ for $j=1, \ldots, n$ such that a certain Schurconvex function $f\left(s_{1}, \ldots, s_{m}\right)$ is minimal (where $s_{i}$ is the sum of elements in i-th row $s_{i}=\sum_{j=1}^{n} a_{\pi_{j}(i), j}$. The most used Schur-convex

- $f_{s q r}\left(s_{1}, \ldots, s_{m}\right)=s_{1}^{2}+s_{2}^{2}+\ldots+s_{m}^{2}$

- $f_{d i f}\left(s_{1}, \ldots, s_{m}\right)=\max \left(s_{1}, \ldots, s_{m}\right)-\min \left(s_{1}, \ldots, s_{m}\right)$

- $f_{\max }\left(s_{1}, \ldots, s_{m}\right)=\max \left(s_{1}, \ldots, s_{m}\right)$

- $f_{s q r}^{\delta}\left(s_{1}, \ldots, s_{m}\right)=\left(s_{1}-\delta\right)^{2}+\ldots+\left(s_{m}-\delta\right)^{2}$ where $\delta=\frac{\left(s_{1}, \ldots, s_{m}\right)}{m}$

In [7] it was shown that MPP is $N P$-hard except two column case for which the polynomial algorithm was found ([2]).

We can gain a generalisation of MPP, if we will not limit ourselves to column permutations and permutations will be allowed by a certain graph (its vertices represent elements of a scheduling matrix, element $a_{i, j}$ is represented by the vertex $v_{i, j}$ ). We will call it regular permutation scheduling on graphs (RPSG). In [3] there were given two ways how to generate needed permutations. We can use:

1. graph of permitted moves,

2. graph automorphisms.

(The graph of permitted moves will be denoted GPM and the related problem GPM-P.)

\section{Graph of permitted moves}

In this section we will continue in our work started in [3]. It is known from this work that MPP can be solved as a special case of GPM-P and if GPM is a complete digraph with loops on every vertex, two-column case can be solved for irregularity measure fsqr as a minimal perfect matching in a certain complete graph. Unfortunately there exist graphs of permitted moves for which two column case can't be solved as a minimal perfect matching in a graph (it was one of the open problems introduced in [3] related to two column GPM-P).

Example 1. In Figure 1 we can see the graph of permitted moves $G_{M}$ and related graph $G$ whose vertex set is the same as the vertex set of $G_{M}$ and vertices $v_{i, j}, v_{k, l}$ are connected by an edge of weight $w=$ $=\left(a_{i, j}+a_{k, l}\right)^{2}$ if and only if there exist directed edges $\left(v_{i, j}, v_{x, y}\right)$ and $\left(v_{k, l}, v_{x, z}\right)$ in $G_{M}(y, z \in\{1,2\} y \neq z)$ it means that $a_{i, j}$ and $a_{k, l}$ could form the row in a new matrix). We can see that for perfect matching with edges $v_{1,1}, v_{1,2}, v_{2,1}, v_{2,2}$ and $v_{3,1}, v_{3,2}$ there doesn't exist any permitted permutation of elements of the matrix $A$ for which elements $a_{1,1}, a_{1,2}$ form some row of new scheduling matrix and elements $a_{2,1}$ $a_{2,2}$ another one.

If we use for two column case another Schur-convex function $f$ and we can transform GPM to a graph in which we find perfect matching then the solution of GPM-P will be perfect matching that minimises $f$.

Example 2. There is given a matrix $A_{3 x 2}=\left(a_{i, j}\right), G P M$ is a complete directed graph with loop on every vertex and fmax $\left(s_{1}, s_{2}, s_{3}\right)=\max \left(s_{1}, s_{2}, s_{3}\right)$ is the irregularity measure. We can solve this problem as finding the perfect matching with minimal $f_{\max }\left(s_{1}, s_{2}, s_{3}\right)$ in a complete graph with the same vertex set as GPM has (where $s_{1}, s_{2}, s_{3}$ are weights of edges used in this matching and edge $\left[v_{i, j}, v_{k, l}\right]$ has weight $\left.a_{i, j}+a_{k, l}\right)$.

\footnotetext{
* Peter Czimmermann

University of Žilina, Faculty of Managements and Informatics, Slovakia, E-mail: petocimo@frcatel.fri.utc.sk
} 
We present an algorithm that finds perfect matching in the graph minimizing the function $f_{\max }$ in graph $G=(V, E)$. (A similar problem is presented in [6] p. 260.)

1. let $n=|V|$ is even and $m=|E|$

2. sort the edges into nondecreasing sequence $S$

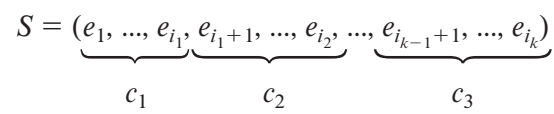

where $i_{k}=m$ and $c_{1}, \ldots, c_{k}$ are weights of the edges

3. find $j \in\{1, \ldots, k\}$ for which $i_{j-1}+1 \leq n / 2 \leq i$

4. if such $j$ does not exist then STOP (there is no perfect matching in $G$ ) else let $r:=j$

5. delete every edge $e$ in $G$ for which its weight $c(e)>c_{r}$ (we obtain a graph

$\left.G^{\prime}=G-\left\{e ; e \in E, c(e)>c_{r}\right\}\right)$

6. for every edge $e=\{u, v\}(u, v \in V)$ of weight $c(e)=c_{r}$ do:

- let $G^{\prime \prime}=G^{\prime}-u-v$

- find perfect matching in $G^{\prime \prime}$

- if $M$ is perfect matching in $G^{\prime \prime}$ then STOP (edge $e$ and edges of $M$ form perfect matching in $G$ for which is function $f_{\max }$ minimal)

7. if $r<k$ then $r:=r+1$ and go to 5 else STOP there isn't any perfect matching in $G$.

It isn't hard to show that the presented algorithm is polynomial. In step 2 it makes $O\left(n^{2} \log n\right)$ steps. An $O\left(n^{5 / 2}\right)$ algorithm is known for finding perfect matching in graphs $[4,6]$ and this algo- rithm must be made $m<n^{2}$ times at most so that our algorithm has complexity $O\left(n^{9 / 2}\right)$.
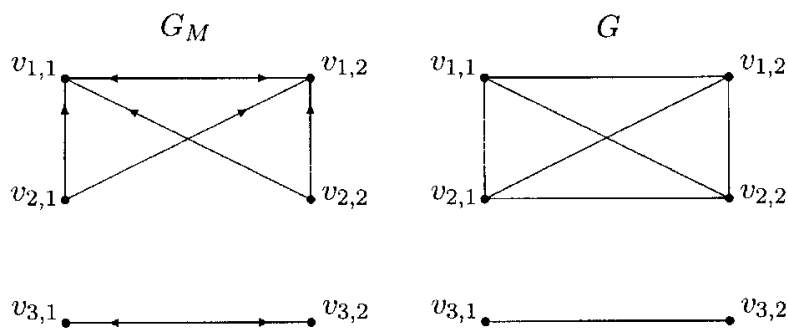

Fig. 1. Graphs GM and $G$

\section{Conclusions}

In this paper we dealt with using the graphs in regular scheduling problems. It seems that perfect matching that is minimal with consideration to some Schur-convex function is an important concept. For most Schur-convex functions $f$ it remains an open problem to find an polynomial algorithm for perfect matching that is minimal with consideration to $f$. Similarly, two column case is unsolved if perfect matching can't be used for representing a convenient solution.

The research of the author is supported by Slovak Scientific Grant Agency under grant NO.1/0490/03.

\section{References}

[1] ČERNÝ, J., KLUVÁNEK, P.: Principles of Mathematical Theory of Transport (in Slovak), VEDA, Bratislava, (1991)

[2] ČERNÝ, J., VAŠEK, K., PEŠKO, Š., PALÚCH, S., ENGELTHALLER, D.: Transport schedulings and their optimization (in Slovak), Research report III-8-9/03, Research Institute of Transport, Žilina, (1986)

[3] CZIMMERMANN P., PEŠKO, Š.: The Regular Permutation Scheduling on Graphs, Journal of Information, Control and Management Systems, vol. 1, (2003)

[4] EVEN, S., KARIV, O.: An $O\left(n^{5 / 2}\right)$ Algorithm for Maximum Matching in General Graphs, Proc. 16th Annual Symp. on Foundations of Computer Science, IEEE, New York, (1975)

[5] PEŠKO, Š., VAŠEK, K.: Optimization of Transport Scheduling (in Slovak), Research report III-8-6/09.3, Research Institute of Transport, Žilina, (1983)

[6] PLESNÍK, J.: Graph Algorithms (in Slovak), VEDA, Bratislava, (1983)

[7] TEGZE, M., VLACH, M.: The Matrix Permutation Problem, Tech. Univ. Graz Bericht 84-54, (1984). 